\title{
EFEITOS DA LACTULOSE NA SAÚDE GASTROINTESTINAL DE FRANGOS DE CORTE EXPERIMENTALMENTE INOCULADOS COM Salmonella entérica SOROVAR Typhimurium
}

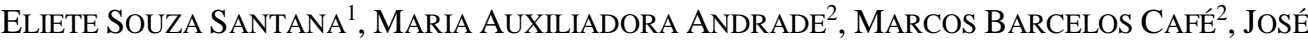 \\ HENRIQUe STRINGHINI ${ }^{2}$, TATIANE MARTINS ROCHA ${ }^{1}$, VALÉRIA DE SÁ JAIME ${ }^{2}$
}

'Pós-Graduandas da Universidade Federal de Goiás, Goiânia, GO, Brasil. elietesouza@ yahoo.com.br ${ }^{2}$ Professores Doutores da Universidade Federal de Goiás, Goiânia, GO, Brasil.

\begin{abstract}
Avaliaram-se os efeitos da lactulose na saúde gastrointestinal de frangos de corte pela aferição do $\mathrm{pH}$ e enumeração de unidades formadoras de colônias (UFCs) de Salmonella Typhimurium e Escherichia coli no inglúvio e ceco de aves inoculadas experimentalmente, via oral, na dose de $5,0 \quad \mathrm{X} \quad 10^{2}$ UFC $/ 0,5 \mathrm{~mL}$ com Salmonella Typhimurium. $\mathrm{O}$ delineamento adotado foi o inteiramente casualizado, utilizando-se 630 pintos, machos, os quais foram distribuídos em seis tratamentos, com sete repetições e 15 aves por unidade experimental. O tratamento 1: grupo controle (placebo); tratamento 2: grupo que recebeu somente a lactulose na água; tratamento 3: grupo que recebeu somente Salmonella Typhimurium; tratamento 4: grupo que recebeu a lactulose e Salmonella Typhimurium simultaneamente no primeiro dia de vida [L (1d) + ST (1d)]; tratamento 5: grupo que recebeu a lactulose 48 horas antes de serem inoculadas com Salmonella Typhimurium [L (1d) + ST (48h)] e tratamento 6: grupo que foi inoculado com Salmonella Typhimurium no primeiro dia e 48 horas
\end{abstract}

depois receberam a lactulose [ST (1d) $+\mathrm{L}(48 \mathrm{~h})]$. Aos dias sete, 14, 21 e 28 uma ave por parcela foi sacrificada e os conteúdos do inglúvio e do ceco foram coletados para a aferição do $\mathrm{pH}$ e contagem de Salmonella Typhimurium e Escherichia coli. Constatou-se que a lactulose determinou redução nos valores $(\mathrm{P}<0,05)$ de $\mathrm{pH}$ nos conteúdos do trato digestório aos sete dias de vida, e esta redução se manteve até 28 dias somente para o inglúvio nos tratamentos que receberam a lactulose, independente do período de inoculação do patógeno. Verificou-se, também, que a lactulose reduziu $(\mathrm{P}<0,05)$ as UFCs de Escherichia coli e de Salmonella Typhimurium no inglúvio aos 21 e 28 dias de vida nos tratamentos em que se administrou a lactulose antes do patógeno. Pode-se concluir que a lactulose altera os valores de $\mathrm{pH}$ do inglúvio e reduza colonização de Salmonella Typhimurium no ceco e as UFCs de Escherichia coli no inglúvio em todo o período experimental.

PALAVRAS-CHAVE: controle, Escherichia coli, $\mathrm{pH}$, unidades formadoras de colônias.

\section{EVALUATION OF LACTULOSE EFFECTS ON INTESTINAL HEALTH OF BROILERS EXPERIMENTALLY INOCULATED WITH Salmonella Typhimurium}

\section{ABSTRACT}

This study was conducted to assess the effects of lactulose on the intestinal lumen by measuring the $\mathrm{pH}$ content of the gastrointestinal tract, and couting the colony forming units (CFU) of Escherichia coli and Salmonella Typhimurium in broilers experimentally and orally inoculated with Salmonella Typhimurium, at the dose of 5.0 X $10^{2} \mathrm{CFU} /$ $\mathrm{mL}$ 0.5. Birds were allotted in a completely randomized design, with 630 day-old male chicks distributed into six 
treatments, with seven replications and 15 birds per experimental unit. Treatment 1: group that did not receive microbial inoculum or lactulose (placebo group); treatment 2: group that received only lactulose (lactulose-L control group); treatment 3: group that received only Salmonella Typhimurium (ST-positive control group); treatment 4: group that received lactulose and Salmonella Typhimurium on the first day of life (L (1) + ST (1)); treatment 5: group that received lactulose 48 hours before the Salmonella Typhimurium (L (1) + ST (48h)); and treatment 6: group that received the Salmonella Typhimurium $48 \mathrm{~h}$ before lactulose (ST (1) + L (48h)). At seven, 14, 21 and 28 days of age, one bird per plot was euthanized and cecum and crop contents were collected for counting of Salmonella Typhimurium and Escherichia coli. Furthermore, $\mathrm{pH}$ of crop and cecum were measured on for the same birds at

KEYWORDS: control, Escherichia coli, pH, UFCs.

\section{INTRODUÇÃO}

Salmonella tem sido apontada como agente de enteroinfecções, veiculado por alimentos e responsável por surtos ou casos esporádicos de toxinfecção alimentar no ser humano. O gênero Salmonella está amplamente distribuído na natureza, possui grande número de reservatórios e apresenta sorotipos inespecíficos quanto aos hospedeiros ${ }^{1}$. As aves, segundo Dawoud et al. ${ }^{2}$, podem apresentar infecção clínica ou subclínica e permanecerem como portadores inaparentes e se tornarem fonte de infecção para humanos, outros animais e meio ambiente.

O controle da salmonelose é um desafio para a avicultura e a saúde pública devido ao surgimento/ressurgimento de sorotipos em áreas diferentes, tanto em países em desenvolvimento como nos desenvolvidos. Os animais portadores inaparentes são elementos epidemiológicos importantes, pois não apresentam sintomas e são difíceis de serem detectados antes ou durante a inspeção sanitária dos animais ${ }^{3}$.

Por essa razão, a indústria avícola brasileira tem adotado vários procedimentos para o controle de salmonelas, dentre os quais se incluem ações no pré-abate com o objetivo de reduzir o número de frangos portadores de Salmonella nesse período. Para isso, a utilização de produtos alternativos, em substituição aos antibióticos promotores de crescimento, constitui uma alternativa viável em relação à segurança alimentar. Dentre esses produtos, pode-se incluir a lactulose, dissacarídeo não digestível, que consiste de uma molécula de seven, 14, 21 and 28 days of age, and blood was collected for liver enzyme evaluation. Lactulose reduced $\mathrm{pH}$ value $(\mathrm{P}<0.05)$ of digestive organs at seven days of life, and this reduction remained until 28 days only in the crop, regardless of the pathogen inoculation, whereas the cecal $\mathrm{pH}$ at 21 and 28 days, did not differ among the other treatments $(\mathrm{P}>0.05)$. We also verified that lactulose reduced $(\mathrm{P}<0.05)$ the $\mathrm{CFU}$ of Escherichia coli and Salmonella Typhimurium in the crop at 21 and 28 days of age in the treatments in which lactulose was administered before the pathogen inoculation. Lactulose changed $\mathrm{pH}$, except cecal $\mathrm{pH}$, and reduced the colonization of Salmonella Typhimurium in the cecum and the number of UFCs of Escherichia coli in the crop during all experimental period.

galactose e uma molécula de frutose (4-O- $\beta$-Dgalactose-D-frutose), usada na medicina humana há mais de 40 anos $^{4}$ com ações hipolipidêmicas, hipoglicêmicas e antimicrobianas e que atua em microrganismos presentes no intestino ${ }^{5}$.

Diante disso, o presente trabalho foi desenvolvido para investigar os efeitos da lactulose na saúde gastrointestinal de frangos de corte, inoculados experimentalmente com Salmonella Typhimurium, pela aferição do $\mathrm{pH}$ e enumeração de unidades formadoras de colônias (UFCs) de Salmonella Typhimurium e Escherichia coli no inglúvio e ceco.

\section{MATERIAL E MÉTODOS}

O experimento foi realizado no Núcleo Experimental de Doenças de Aves da Escola de Veterinária e Zootecnia da Universidade Federal de Goiás. O protocolo experimental utilizado neste estudo foi aprovado pelo Comitê de Ética em Pesquisa da UFG, sob o $n^{\circ} 103 / 09$, e está de acordo com os Princípios Éticos na Experimentação Animal, adotado pela Sociedade Brasileira de Ciência em Animais de Laboratório (SBCAL).

Foram utilizados 630 pintos da linhagem Cobb, machos, de um dia de idade, os quais foram divididos em seis tratamentos, com sete repetições cada, em grupos de 15 aves por unidade experimental, em 42 unidades experimentais. Os tratamentos foram assim constituídos:

1: grupo controle (placebo);

2: grupo de aves inoculadas diretamente no inglúvio no primeiro dia de vida, com solução 
contendo Salmonella Typhimurium - controle positivo - [ST];

3: grupo que recebeu água adicionada com lactulose do primeiro aos 14 dias de vida - controle da Lactulose - [L];

4: grupo de aves inoculadas diretamente no inglúvio no primeiro dia de vida, com solução contendo Salmonella Typhimurium e, 48 horas depois, a lactulose foi fornecida na água de bebida até 14 dias de vida da ave [ST (1d) + L(48h)];

5: grupo de aves que recebeu a água com lactulose desde o primeiro dia de vida na mesma dose do controle e, 48 horas depois, foram inoculadas diretamente no inglúvio com solução contendo Salmonella Typhimurium $[\mathrm{L}(1 \mathrm{~d})+$ ST(48)];

6: grupo de aves que recebeu diretamente no inglúvio a solução contendo Salmonella Typhimurium e a água com lactulose do primeiro até aos 14 dias de vida [ST(1d) + L(1d)]

O inóculo foi preparado segundo Fernández et al. $^{6}$ na forma de solução salina tamponada a $0,85 \%$, contendo, aproximadamente, 5,0 × $10^{2}$ UFC/0,5 mL de Salmonella Typhimurium. A Salmonella Typhimurium foi isolada de amostras oriundas de frangos de corte cedidas pelo Laboratório de Bacteriologia da Escola de Veterinária e Zootecnia da Universidade Federal de
Goiás e tipificadas pelo laboratório FIOCRUZ (RJ). As aves inoculadas com o patógeno foram alojadas separadamente dos grupos controle sob as mesmas condições ambientais. A lactulose foi fornecida na dosagem de $0,023 \mathrm{~mL} / \mathrm{g}$ de peso corporal, sendo que essa dose foi determinada em pesquisas desenvolvidas por Santana et al. ${ }^{7}$.

Aos sete, 14, 21 e 28 dias de idade, uma ave por parcela foi sacrificada por deslocamento cervical e eutanasiada após jejum alimentar de duas a três horas. Os conteúdos do ceco e inglúvio foram avaliados quanto ao $\mathrm{pH}$, segundo Silva et al. ${ }^{8}$, e submetidos à quantificação de UFCs de E. coli e Salmonella Typhimurium, de acordo com Brasil ${ }^{9}$.

Os dados quantitativos de $\mathrm{pH}$ dos inglúvios e dos cecos, bem como as médias da enumeração das UFCs, expressas em log, de Salmonella Typhimurium e E. coli foram submetidos à análise de variância (ANOVA) e as médias foram comparadas pelo teste de Tukey a 5\%, sendo utilizado o procedimento general linear model (GLM) do pacote estatístico do $\mathrm{SAS}^{10}$.

\section{RESULTADOS}

Os valores de $\mathrm{pH}$ do inglúvio e ceco foram influenciados $(\mathrm{P}<0,05)$ pelos tratamentos (Tabela $1)$.

Tabela 1- Resultados das aferições de pH do inglúvio e ceco de frango de corte aos sete, 14, 21 e 28 dias de vida, recebendo lactulose (L) e/ou Salmonella Typhimurium (ST)

\begin{tabular}{|c|c|c|c|c|}
\hline & \multicolumn{4}{|c|}{ Inglúvio } \\
\hline Tratamento & 7 dias & 14 dias & 21 dias & 28 dias \\
\hline Placebo & $6,64 \mathrm{~A}$ & $6,66^{\mathrm{a}}$ & $7,10 \mathrm{~A}$ & $6,65 \mathrm{~A}$ \\
\hline Controle positivo $[\mathrm{ST}]$ & $5,27 \mathrm{BC}$ & $6,25 \mathrm{AB}$ & $6,06 \mathrm{~B}$ & $4,83 \mathrm{~B}$ \\
\hline Controle lactulose [L] & $4,82 \mathrm{C}$ & $6,34 \mathrm{AB}$ & $6,44 \mathrm{AB}$ & $6,61 \mathrm{~A}$ \\
\hline$[\mathrm{ST}(1 \mathrm{~d})+\mathrm{L}(1 \mathrm{~d})]$ & $6,44 \mathrm{AB}$ & $5,58 \mathrm{~B}$ & $5,59 \mathrm{BC}$ & $5,17 \mathrm{~B}$ \\
\hline$[\mathrm{L}(1 \mathrm{~d})+\mathrm{ST}(48 \mathrm{~h})]$ & $5,34 \mathrm{BC}$ & $5,61 \mathrm{AB}$ & $4,35 \mathrm{D}$ & $5,50 \mathrm{AB}$ \\
\hline$[S T(1 d)+L(48 h)]$ & $5,63 \mathrm{ABC}$ & $6,15 \mathrm{AB}$ & $4,93 \mathrm{DC}$ & $5,08 \mathrm{~B}$ \\
\hline C. V. $(\%)$ & 13,12 & 11,01 & 10,03 & 13,90 \\
\hline \multirow[t]{2}{*}{$\mathrm{P}$} & 0,0003 & 0,0308 & 0,0001 & 0,0001 \\
\hline & \multicolumn{4}{|c|}{ Ceco } \\
\hline Tratamento & 7 dias & 14 dias & 21 dias & 28 dias \\
\hline Placebo & $7,20 \mathrm{~A}$ & 6,92 & 6,52 & 6,83 \\
\hline Controle positivo [ST] & $6,58 \mathrm{AB}$ & 6,71 & 6,69 & 6,46 \\
\hline Controle lactulose [L] & $5,97 \mathrm{~B}$ & 6,68 & 6,68 & 6,95 \\
\hline [ST (1d) + L (1d)] & $6,45 \mathrm{AB}$ & 6,56 & 6,94 & 6,69 \\
\hline$[\mathrm{L}(1 \mathrm{~d})+\mathrm{ST}(48 \mathrm{~h})]$ & $6,63 \mathrm{AB}$ & 6,75 & 6,77 & 6,64 \\
\hline$[\mathrm{ST}(1 \mathrm{~d})+\mathrm{L}(48 \mathrm{~h})]$ & $5,85 \mathrm{~B}$ & 6,84 & 6,87 & 6,58 \\
\hline C. V. $(\%)$ & 10,48 & 7,30 & 5,40 & 4,49 \\
\hline $\mathrm{P}$ & 0,0085 & 0,8175 & 0,3589 & 0,0571 \\
\hline
\end{tabular}

*Letras diferentes na mesma coluna diferem entre si pelo teste de Tukey $(\mathrm{P}<0,05)$. 
No inglúvio, os menores valores de $\mathrm{pH}$ foram obtidos com o tratamento [L] aos sete dias, [ST (1d) + L (1d)] aos 14 dias, [L (1d) + ST (48h)] aos 21 dias e [ST] e [ST (1d) + L (1d)] aos 28 dias de idade. É possível observar pronunciada redução nos valores de $\mathrm{pH}$ do inglúvio das aves que foram inoculadas com Salmonella Typhimurium e lactulose no primeiro dia de vida, em relação ao placebo, após 14 dias de vida. Com relação ao $\mathrm{pH}$ do ceco, houve redução somente com os tratamento [L] e [ST (1d) + L (48h)] aos sete dias.

Com base nos resultados obtidos nas diferentes idades, observou-se que as aves que receberam a lactulose 48 horas antes ou após serem desafiadas com Salmonella Typhimurium apresentaram a maior redução do $\mathrm{pH}$ do inglúvio em relação aos demais grupos, os quais também apresentaram valores de $\mathrm{pH}$ menores do que o grupo placebo. Por outro lado, o $\mathrm{pH}$ cecal não mostrou diferença $(P>0,05)$ entre os diferentes tratamentos.

A análise dos dados obtidos aos 28 dias de idade (Tabela 1) mostraram que as aves que compuseram os tratamentos placebo, controle da lactulose [L] e o que recebeu a lactulose 48 horas antes da inoculação do patógeno [L (1d) + ST (48h)] obtiveram maiores valores de $\mathrm{pH}$ para o inglúvio, enquanto foram observados valores menores em aves inoculadas com Salmonella Typhimurium no primeiro dia de vida. Além disso, notou-se que, nas amostras do ceco, o grupo controle da lactulose apresentou maior valor de $\mathrm{pH}$ e foi semelhante ao do grupo placebo, porém sem apresentar diferença significativa $(\mathrm{P}>0,05)$.

Os valores médios de contagem de UFCs, expressos em $\log$, de $E$. coli no inglúvio nas diferentes idades avaliadas, com exceção da avaliação realizada aos 21 dias, apresentaram diferença significativa $(\mathrm{P}<0,05)$ para UFC / $\mathrm{mL}$ de conteúdo do inglúvio em relação ao placebo (Tabela 2).

Observou-se, ainda, que os menores valores de UFCs do inglúvio foram encontrados nos grupos que receberam somente a lactulose [L] e naqueles inoculados experimentalmente com Salmonella Typhimurium e tratados com lactulose até os 14 dias do experimento - [ST (1d) + L (1d)] e [ST (1d) + L(48h)]. Verificou-se, também, reduzida contagem ou mesmo ausência de E. coli no inglúvio, no grupo controle da lactulose [L] em todas as idades avaliadas. Já para a contagem de $E$. coli no ceco, não se observou diferença $(\mathrm{P}>0,05)$ entre os diversos grupos estudados, durante todos os períodos analisados.

Tabela 2-Valores médios da contagem de Unidades Formadoras de Colônias (UFCs), expressa em log, de $E$. coli no inglúvio e no ceco de frangos de corte inoculados com lactulose (L) e/ou Salmonella Typhimurium (ST) aos 14, 21 e 28 dias de idade

\begin{tabular}{lccc}
\hline \multirow{2}{*}{ Tratamentos } & \multicolumn{3}{c}{ Inglúvio } \\
\cline { 2 - 4 } & 14 dias & 21 dias & 28 dias \\
\hline Placebo & $4,44 \mathrm{~A}$ & 4,42 & $3,31^{\mathrm{a}}$ \\
Controle positivo [ST] & $2,91 \mathrm{AB}$ & 3,34 & $2,93 \mathrm{AB}$ \\
Controle lactulose [L] & $0,84 \mathrm{C}$ & 0,00 & $1,42 \mathrm{~B}$ \\
{$[\mathrm{ST}(1 \mathrm{~d})+\mathrm{L}(1 \mathrm{~d})]$} & $1,22 \mathrm{BC}$ & 3,08 & $2,60 \mathrm{AB}$ \\
{$[\mathrm{L}(1 \mathrm{~d})+\mathrm{ST}(48 \mathrm{~h})]$} & $0,29 \mathrm{C}$ & 2,85 & $1,64 \mathrm{AB}$ \\
{$[\mathrm{ST}(1 \mathrm{~d})+\mathrm{L}(48 \mathrm{~h})]$} & - & 2,11 & $2,06 \mathrm{AB}$ \\
\hline $\mathrm{P}$ & $<0,0001$ & 0,0925 & 0,0302 \\
\hline Tratamentos & & Ceco & 28 dias \\
\hline Placebo & 14 dias & 21 dias \\
Controle positivo [ST] & 4,57 & 5,83 & 5,72 \\
Controle lactulose [L] & 5,40 & 5,94 & 6,13 \\
{$[\mathrm{ST}(1 \mathrm{~d})+\mathrm{L}(1 \mathrm{~d})]$} & 6,48 & 5,93 & 6,01 \\
{$[\mathrm{~L}(1 \mathrm{~d})+\mathrm{ST}(48 \mathrm{~h})]$} & 6,45 & 6,95 & 6,63 \\
{$[$ ST (1d) + L (48h)] } & 4,10 & 6,49 & 6,12 \\
\hline P & 6,25 & 6,82 & 6,21 \\
\hline
\end{tabular}

(-) Ausência; Médias seguidas de letras diferentes na mesma coluna diferem entre si pelo teste de Tukey $(\mathrm{P}<0,05)$.

Analisando-se os resultados referentes à contagem de Salmonella Typhimurium dos conteúdos dos inglúvios e ceco (Tabela 3 ), verificou-se que a contagem de UFCs da Salmonella Typhimurium aos 14 dias no grupo que recebeu a lactulose 48 horas antes do patógeno [L (1d) + ST (48h)] apresentou 
menor contagem, com diferença significativa $(\mathrm{P}<0,05)$ em relação aos demais tratamentos. Destacase, ainda, que os grupos que receberam o patógeno antes da lactulose [ST (1d) + L (48h)], assim como o controle positivo, apresentaram uma contagem mais elevada de UFCs.

Observou-se, também, que, aos 21 dias, as UFCs de Salmonella Typhimurium obtidas do inglúvio se mantiveram mais altas no controle positivo [ST] em relação aos demais tratamentos. Destaca-se, ainda, que as UFCs do conteúdo cecal nos grupos que receberam simultaneamente o patógeno e lactulose [ST (1d) + L (1d)] e o patógeno e posteriormente a lactulose [ST (1d) + L (48h)], não apresentaram diferenças $(\mathrm{P}>0,05)$ do grupo controle positivo (Tabela 3).

Tabela 3- Valores médios de unidades formadoras de colônias (UFCs) de Salmonella Typhimurium, expressas em log, no inglúvio e no ceco de frangos de corte recebendo lactulose (L) e/ou Salmonella Typhimurium (ST) aos 14,21 e 28 dias de idade

\begin{tabular}{lccc}
\hline \multirow{2}{*}{ Tratamentos } & \multicolumn{3}{c}{ Inglúvio } \\
\cline { 2 - 4 } & 14 dias & 21 dias & 28 dias \\
\hline Placebo & - & - & - \\
Controle positivo [ST] & $1,11^{\mathrm{a}}$ & $1,73 \mathrm{~A}$ & $1,54 \mathrm{~A}$ \\
Controle lactulose [L] & - & - & - \\
{$[\mathrm{ST}(1 \mathrm{~d})+\mathrm{L}(1 \mathrm{~d})]$} & - & - & $1,43^{\mathrm{a}}$ \\
{$[\mathrm{L}(1 \mathrm{~d})+\mathrm{ST}(48 \mathrm{~h})]$} & - & $0,53 \mathrm{~B}$ & - \\
{$[\mathrm{ST}(1 \mathrm{~d})+\mathrm{L}(48 \mathrm{~h})]$} & $0,38 \mathrm{~B}$ & - & $0,10 \mathrm{~B}$ \\
\hline P & $<0,0001$ & Ceco & $<0,0001$ \\
\hline Tratamentos & & 21 dias & 28 dias \\
\hline Placebo & 14 dias & - & - \\
Controle positivo [ST] & - & $0,10 \mathrm{~B}$ & $0,10 \mathrm{~B}$ \\
Controle lactulose [L] & $3,24^{\mathrm{a}}$ & - & - \\
{$[\mathrm{ST}(1 \mathrm{~d})+\mathrm{L}(1 \mathrm{~d})]$} & - & $1,00 \mathrm{~A}$ & $1,00 \mathrm{~A}$ \\
{$[\mathrm{~L}(1 \mathrm{~d})+$ ST (48h)] } & - & - & - \\
{$[$ ST (1d) + L (48h)] } & - & $1,00 \mathrm{~A}$ & $1,00 \mathrm{~A}$ \\
\hline P & $2,25 \mathrm{~B}$ & $<0,0001$ & $<0,0001$ \\
\hline
\end{tabular}

(-) Ausência; Médias seguidas de letras diferentes na mesma coluna diferem entre si pelo teste de Tukey $(\mathrm{P}<0,05)$.

Analisando-se, ainda, a Tabela 3, observa-se que, aos 28 dias, os valores de UFCs no inglúvio foram maiores para o controle positivo, que se mostrou estatisticamente diferente dos grupos que receberam o patógeno associado à lactulose - [ST (1d) + L (1d)], [L (1d) + ST (48h)] e [ST (1d) + L (48h)]. No ceco, menor contagem foi constatada para o controle positivo, que foi diferente $(\mathrm{P}<0,05)$ dos grupos que receberam a Salmonella Typhimurium e a lactulose [ST (1d) + L (1d)] e a lactulose e posteriormente o patógeno [L (1d) + ST (48h)].

\section{DISCUSSÃO}

Neste estudo, enquanto a lactulose foi fornecida, associada ou não Salmonella Typhimurium, ocorreu redução do $\mathrm{pH}$ no inglúvio em todo o período experimental e até os sete dias no ceco. De acordo com Konstantinov et al. ${ }^{11}$, a lactulose é um dissacarídeo, composto por uma molécula de galactose e uma molécula de frutose (4-O- $\beta$-D-
galactose-D-frutose), considerada um isômero da lactose, que leva à diminuição do desenvolvimento de diversas bactérias intestinais, patogênicas ou não, pelo aumento da quantidade de ácido láctico com consequente redução do $\mathrm{pH}$.

Destaca-se que, no mesmo período em que se observou uma redução de $\mathrm{pH}$ no ceco, ocorreu uma reduzida contagem ou mesmo ausência de $E$. coli no mesmo órgão nos grupos que receberam a lactulose. Isso permite inferir que a redução de $\mathrm{pH}$ contribuiu para a diminuição de E. coli. Tais resultados estão respaldados nos dados experimentais obtidos por Mathew et al. ${ }^{12}$, que adicionaram $1 \%$ de galactanas, e por Gebbink $^{13}$, que adicionaram $5 \%$ de frutooligossacarídio (FOS) em dietas para leitões recém desmamados, demonstrando a ação efetiva desses compostos no aumento na população de bactérias lácticas, na redução do $\mathrm{pH}$ e na diminuição na contagem de E. coli.

Não observou crescimento de UFCs de E. coli nos grupos inoculados experimentalmente, aos14 
dias, com Salmonella Typhimurium e tratados com a lactulose. A inibição do crescimento da $E$. coli pode ser atribuído à competição entre os microrganismos presentes no inglúvio, havendo, por exclusão, a modificação na microbiota transiente (residente temporária) e mesmo a indígena (colonização permanente) do inglúvio.

Por outro lado, o $\mathrm{pH}$ cecal não mostrou diferenças $(\mathrm{P}>0,05)$ entre os distintos tratamentos aos 14 e 21 dias. Esperavam-se valores menores de $\mathrm{pH}$ pelo efeito da lactulose, tendo em vista que, inicialmente, esta alteração ocorreu e, consequentemente, haveria aumento de bactérias benéficas produtoras de ácidos orgânicos.

Pondera-se que outros fatores como dieta, renovação celular, temperatura e privação de alimentos poderiam interferir nesse processo. Hipóteses prováveis seriam a dose, o veículo e o período de administração da substância, que talvez não tenham sido suficientes para modificar ou promover essa mudança de $\mathrm{pH}$ até 21 dias do experimento. Outra possibilidade seria um mecanismo de ação da lactulose diferente do conhecido, pois de acordo com Martín-Peláez et al. ${ }^{14}$, nem sempre a ingestão de compostos com potencial ação prebiótica causa mudanças no $\mathrm{pH}$ cecal e isso pode estar relacionado com a população microbiana da espécie animal e a ingestão da quantidade do produto adicionado à alimentação.

Aos 28 dias, constataram-se os maiores valores de $\mathrm{pH}$ do inglúvio e ceco para os tratamentos que receberam a droga e o placebo. O comportamento da Salmonella Typhimurium isoladamente, quando inoculada simultaneamente com a lactulose, ou 48 horas após a inoculação da Salmonella propiciou discreto efeito nas condições luminais do intestino, propiciando maior desenvolvimento da Salmonella. Respaldados pelos estudos realizados por Freitas Neto $^{15}$, que afirmou que o trato entérico das aves tem complexidade ainda não totalmente conhecida, mas a composição nutricional da alimentação fornecida, o pH de cada porção do intestino, a tensão de oxigênio no intestino delgado e dióxido de carbono no ceco são fatores que podem modificar a microbiota intestinal.

Não se observaram diferenças $(\mathrm{P}>0,05)$ na quantificação de Escherichia coli no ceco. Esse resultado não pode ser atribuído às mudanças de $\mathrm{pH}$, pois os valores aos 14 e 21 dias não se modificaram, e aos 28 dias, verificou-se o maior valor de $\mathrm{pH}$ no controle da lactulose [L], que foi semelhante ao grupo placebo. Tais valores foram diferentes dos resultados obtidos por Stanley et al. ${ }^{16}$, que observaram significativa redução na contagem de coliformes totais $\left(2 \log _{10}\right)$ em cecos de frangos de corte que receberam $0,2 \%$ de lactulose na dieta. No entanto, outros fatores como a concentração da droga, a ração que foi mudada devido à fase experimental e talvez a um mecanismo de ação desconhecido da lactulose podem ter contribuído para essas diferenças.

Acrescenta-se que E. coli, integrante do trato gastrointestinal das aves, não tem seu papel totalmente elucidado nas atividades intestinais, sendo encontrada em concentrações acima de $10^{6}$ UFCs por grama de fezes. Embora seu papel não esteja totalmente definido, $10-15 \%$ dessa população pode ser considerada potencialmente patogênica ${ }^{17}$.

A quantificação de UFCs de Salmonella Typhimurium aos 14 dias do conteúdo do inglúvio, assim como do conteúdo do ceco, apresentou diferenças $(\mathrm{P}<0,05)$ em relação aos demais tratamentos. Verifica-se que os grupos que receberam o patógeno e simultaneamente a lactulose [ST (1d) + L (1d)], e a lactulose 48 horas antes do desafio por Salmonella Typhimurium [L (1d) + ST (48h)] apresentaram ausência de UFCs do agente inoculado. Isso permite especular que a lactulose reduziu a colonização por Salmonella Typhimurium no inglúvio e no ceco. Destaca-se que o ceco é o local onde a Salmonella encontra condições para se desenvolver com mais facilidade. Essa característica, conforme McHan et al. ${ }^{18}$, relaciona-se com receptores específicos no órgão e com a fisiologia do peristaltismo cecal, que possibilita maior tempo de permanência do bolo alimentar. Provavelmente, o fornecimento da lactulose aumentou o peristaltismo cecal, determinando menor tempo de permanência do bolo alimentar e, portanto, menor colonização pela bactéria inoculada. Resultados semelhantes foram encontrados por Gabriel et al. ${ }^{19}$, que relataram que a adição de altos níveis de mananoligossacarídeos fosforilado $(0,40 \%)$ na dieta de frangos jovens desafiados com Salmonella reduziu as contagens de bactérias no ceco.

Com o avançar da idade, a recuperação da Salmonella Typhimurium nos conteúdos do inglúvio no grupo controle positivo [ST] não apresentou diferença estatística $(\mathrm{P}>0,05)$ em relação ao grupo que recebeu o patógeno e lactulose [ST(1d) $+\mathrm{L}$ (1d)]. Por outro lado, a contagem de UFCs de Salmonella Typhimurium no ceco foi menor para o controle positivo $[\mathrm{ST}]$ e diferente $(\mathrm{P}<0,05)$ dos grupos que receberam Salmonella Typhimurium e a lactulose [ST (1d) + L (1d)] e a lactulose e posteriormente o patógeno [L (1d) + ST (48h)]. O que pode ser destacado é que a idade, assim como a lactulose, enquanto fornecida, foram fatores importante na redução da colonização no ceco. Provavelmente, a lactulose, enquanto era ingerida, impediu a colonização do patógeno, uma vez que a bactéria foi eliminada nas fezes das aves inoculadas que contaminaram o ambiente de alojamento, as quais se infectaram mais após a retirada do prebiótico. Isso 
sugere que o dissacarídeo foi capaz de bloquear a aderência do patógeno e evitar a colonização enquanto presente no lúmen intestinal. Essa justificativa encontra respaldo nas observações feitas por Kim et al. ${ }^{20}$, que relataram a possibilidade de as bactérias se ligarem a um açúcar e não à mucosa intestinal e passarem com a ingesta sem causar problemas digestivos para os animais.

Mediante os resultados obtidos, pode-se inferir que a lactulose reduziu ou impediu a colonização do ceco pela Salmonella ou, ainda, estimulou seletivamente o crescimento bacteriano, ativando o metabolismo de bactérias benéficas do trato gastrointestinal. Tais ações foram parcialmente discutidas por Santin et al. ${ }^{21}$ e Kim et al. ${ }^{20}$, que relataram que mananoligossacarídeos possuem características específicas, que permitem reduzir a colonização de patógenos no organismo. Portanto, além dos benefícios da saúde do próprio animal, também existe o benefício na segurança dos alimentos pela redução de contaminação de carcaças.

\section{CONCLUSÕES}

Constata-se que a lactulose altera os valores de $\mathrm{pH}$ do inglúvio e ceco, assim como reduz a colonização de Salmonella Typhimurium no ceco e as UFCs de Escherichia coli no inglúvio em todo período experimental.

\section{REFERÊNCIAS}

1. Maianali, C, Mcfall, ME, King, RK. Validation of a realtime polymerase chain reaction assay for the detection of Salmonella in crops of broiler chickens. Poultry Science. 2011, 90(1): 660-64.

2. Dawoud, TM., Hererra, P, Hanning, I, Kwon, YM, Ricke, SC In vitro invasion of laying hen ovarian follicles by Salmonella Enteritidis strains. Poultry Science. 2011, 90(1):1134-37.

3. Tessari, ENC, Cardoso, ALSP., Castro AGM, Zanatta, GF, Kanashiro, AM Incidência de Salmonella sp. em pintos de corte recém-nascidos. Arq. Inst. Biol., 2012, 70(3): 27981 .

4. Schumann, R. Clinical Medical and technological propertie of lactulose. An Update. Eur. J. Nutr. 2002, 41(1): $117-25$.

5. Holz, PH, Middleton, DR. Prospective Study. Medical Surgery. 2005, 15(1): 4-6.

6. Fernández, A, Lara, C, Loste, A, Calvo, S, Marca, MC. Control of Salmonella Enteritidis phage type 4 experimental infection by fosfomycin in newly hatched chicks. Comp. Immunol. Microb. 2001, 24(2): 207-16.

7. Santana, ES, Andrade, MA, Rocha, TM, Stringhini, JH,
Café, MB, Jayme, VS, Barnabé, ACS, Alcântara, JB. Performance of broilers experimentally inoculated with Salmonella Typhimurium and fed diets with addition of lactulosis. Rev. Bras. Zootecn. 2012, 41(8): 1884-89.

8. Silva, EN, Teixeira, AS, Fialho, ET, Bertechini, AG, Souza, PRI. Efeitos dos probióticos e antibióticos sobre as vilosidades e $\mathrm{pH}$ do trato gastrointestinal de frangos de corte. Cienc. agrotec. 2000, 24(2): 163-73.

9. Brasil. Ministério da Agricultura, Pecuária e Abastecimento. Secretaria da Defesa Agropecuária. Instrução Normativa n. 62 de 26/08/2003. Publicada em 18/09/2003. Métodos analíticos oficiais para análises microbiológicas para controle de produtos de origem animal e água. Brasília. DF: MAPA, 2008. 123 p. Disponível em: http://www.agricultura.gov.br/arq_editor/file/Aniamal/Lab orat\%C3\%B3rios/ESCOPOS/Escopo\%20MIC\%20$\% 20$ Vers\%C3\%A3o\%20de\%2013-01-

2012\%20p_\%20site.pdf. Data de acesso: 27/09/2010.

10. SAS Institute. SAS (Statistical Analysis System). User's Guide: Statistics. Cary, NC: SAS Institute INC, 2000 .

11. Konstantinov, SR, Awati, A, Smidt, H, Williams, BA, Akkermans, AD, De WM. Specific response of a novel and abundant Lactobacillus amylovorus Vos, like phylotype to dietary prebiotics in the guts of weaning piglets. Appl. Environ. Microbiol. 2004, 70(1): 3821-30.

12. Mathew, AG, Sutton, AL, Scheidt, AB, Patterson, JA, Kelly DT, Meyerholtz, K. A. Effect of galactan on selected microbial populations and $\mathrm{pH}$ and volatile fatty acids in the ileum of the weanling pig. J. Anim. Sci. 1993, 71(6): 150309.

13. Gebbink, GAR. Effects of addition of frutooligosaccharide (FOS) and sugar beet pulp to weanling pig diets on performance, microflora and intestinal health. Acesso em 12 de de setembro 2009. Online. Disponível em: http://www.ansc.purdue.edu/swine/swineday/sday99/9.pdf

14. Martín-Peláez, S, Costabile, A, Hoyles, L, Rastall, RA, Gibson, GR, La Ragione, RM, Woodward, MJ, Mateu, E, Martín-Orúe, SM. Evaluation of the inclusion of a mixture of organic acids or lactulose into the feed of pigs experimentally challenged with Salmonella Typhimurium, Vet. Microbiol. 2009, 9(61): 1-29.

15. Freitas Neto, OC, Arroyave, WH, Alessi, AC, Fagliari, JJ, Berchieri Júnior, A. Salmonella Gallinarum: Clinical, anatomophatological and haematological studies. Poultry Science. 2007, 9(3): 133-41.

16. Stanley, VG, Gray, C, Chukwu, H. Effects of lactose and Bio-MOS in dietary application on growth and total coliform bacteria reduction in broiler chicks. Poultry Science. 1996, 75(1): 61.

17. Jeffrey, JS, Nolan, LK, Tonooka, KH, Wolfe, S, Giddings, CW, Horne, SM, Foley, SL, Lynne, AM, Ebert, JO, Elijah, LM, Bjorklund, G, Pfaff-Mcdonough, SJ, 
Singer, RS, Doetkott, C. Virulence factors of Escherichia coli from cellulitis or colisepticemia lesions in chickens. Avian Diseases. 2002, 46(1): 48-52.

18. Mchan, CNA, Blankenship, LC, Bailey, JS. In vitro attachament of Salmonella Typhimurium to chick ceca exposed to select carbohydrates. Avian Diseases. 1989, 33(1): 340-44.

19. Gabriel, I, Mallet, S, Leconte, M, Travel, A, Lalles, JP. Effects of whole wheat feeding on the development of the digestive tract of broiler chickens. Anim. Feed. Sci. Tech. 2008, 14(2): 144-62.
20. Kim, GB, Seo, YM, Kim, CH, Paik, IK. Effect of dietary prebiotic supplementation on the performance, intestinal microflora, and immune response of broilers. Poultry Science. 2011, 90(2): 75-82.

21. Santin, E, Maiorka, A, Macari, M, Grecco, M, Sanchez, JC, Okada, MT, Myasaka, AM. Performance and intestinal mucosa development of broiler chickens fed diets containing Saccharomyces cerevisiae cell wall. J. Appl. Poultry Res. 2001, 10(2), p. 236-44. 